\title{
Genese, Formierung, Ausdifferenzierung und Durchbruch des Journalismus in Deutschland
}

\author{
Thomas Birkner
}

Bislang wurde deutsche Journalismusgeschichte anhand von Dieter Paul Baumerts „Die Entstehung des deutschen Journalismus" aus dem Jabr 1928 in eine präjournalistische Periode sowie Perioden des korrespondierenden, des schriftstellerischen und letztlich des redaktionellen Journalismus unterteilt. Der vorliegende Beitrag stellt auf der Basis einer interdisziplinären Integration von Journalismusforschung und Gesellschaftsgeschichte komplementär zu Baumert eine neue Phaseneinteilung vor, welche auch die gesamtgesellschaftlichen Rabmenbedingungen berücksichtigt. Auf den Ebenen von journalistischen Institutionen, Akteuren und Aussagen werden so eine Phase der Genese von 1605 bis 1848, eine Phase der Formierung von 1849 bis 1873, eine Phase der Ausdifferenzierung von 1874 bis 1900 und schließlich der Durchbruch des modernen Journalismus in Deutschland im ersten Jahrzehnt des 20. Jahrhunderts herausgearbeitet. Hierzu wird bei der Rekonstruktion dieses Durchbruchs in der deutschen Journalismusgeschichte auch auf die zeitgenössische Praktikerliteratur zurückgegriffen.

Schlagwörter: Journalismusgeschichte, Sozialgeschichte, Journalismustheorie, Praktikerliteratur

\section{Journalismusgeschichte als Sozialgeschichte}

„Insgesamt“, so Siegfried Weischenberg (2005: 134), „ist die Geschichte insbesondere des deutschen Journalismus nach wie vor unzureichend untersucht." Diesen Befund teilen unter anderem Walter Hömberg (1987: 621), Bernd Blöbaum (1994: 84ff.), Hannes Haas (1999: 99), Susanne Kinnebrock (2005: 103) und Hartwig Gebhardt (2007: 11). Häufig wird in diesem Zusammenhang kritisch - aber alternativlos - auf die 1928 in Die Entstebung des deutschen Journalismus von Dieter Paul Baumert entwickelte Periodisierung hingewiesen (vgl. u. a. Groth 1962: 4f.; Hömberg 1987: 624; Pürer/Raabe 2002: 409ff.; Pürer 2003: 110ff.; Kinnebrock 2005: 103; Weischenberg 2005: 134). Baumert unterschied damals nach einer präjournalistischen Periode, Perioden des korrespondierenden und schriftstellerischen Journalismus, sowie letztlich des redaktionellen Journalismus. Rudolf Stöber (2005a: 15) hat angemerkt, dass die korrespondierende wie schriftstellerische Periode ebenfalls präjournalistisch seien.

Durchaus eingängig war Baumerts Einteilung anhand der jeweils dominierenden handelnden Personen, also der Korrespondenten, Schriftsteller und Redakteure, denn er wollte „die persönliche Seite in den Vordergrund“ (Baumert 1928: 16) stellen. Neben der wichtigen Beschäftigung mit Personen aber gilt es, ihre Texte sowie die Organisationen, in deren Strukturen diese entstehen und in denen Journalisten arbeiten, als endogene Faktoren der Geschichte des Journalismus, einzubeziehen. Doch Stöber (2005a: 22ff.) hat die Kommunikationswissenschaft darauf hingewiesen, dass rein endogene Erklärungsversuche unzureichend seien. Gleichsam hat Pierre Bourdieu (2005: 33) wiederum die Soziologie ermahnt, dass es eine rein exogene Herangehensweise ebenso wenig ermögliche, Journalismus zu verstehen.

Schon Baumert hatte, allerdings ohne diesem Anspruch gerecht zu werden, sein Werk eine sozialgeschichtliche Studie genannt. Später forderte dann Kurt Koszyk (1989: 46; vgl. auch Haas 1999: 100) „Kommunikationsgeschichte als Sozialgeschichte“. Auch 
Manfred Rühl (1992: 131, vgl. ders. 1980) erklärte, dass Journalismus „stets abhängig von einem soziohistorisch zu bestimmenden Gesellschaftssystem“ sei. Wir können also Journalismus, verstanden als komplex ausdifferenziertes Selbstbeobachtungssystem der ebenso komplex ausdifferenzierten Gesellschaft, nur innerhalb der ihn gesellschaftlich beeinflussenden exogenen Faktoren untersuchen. Damit tritt die Gesellschaftsgeschichte an die Seite der kommunikationswissenschaftlichen Journalismusforschung, um die Rahmenbedingungen für die Entstehung des modernen Journalismus erklären zu können.

Hierzu wird zunächst die Gesellschaft in Anlehnung an Max Weber und Hans-Ulrich Wehler (1987a: 9ff.) unterteilt in die Dimensionen Sozialstruktur und Kultur, Wirtschaft und Technologie sowie Politik und Recht, wobei die jeweiligen Entwicklungen stets auf den Journalismus fokussiert werden. In der Dimension von Sozialstruktur und Kultur stehen dabei das Bevölkerungswachstum und die Ausbreitung der Alphabetisierung im Vordergrund. In der wirtschaftlichen und technologischen Dimension geht es vor allem um die Ökonomisierung des Pressewesens und die technologischen Weiterentwicklungen, ohne dabei ein oft betontes - aber zu Recht bereits von Knut Hickethier (2003) abgelehntes - „medientechnisches Apriori“ zu unterstellen. Auch Wehler (1987a: 9; vgl. Luhmann 2005 [1987]: 36) verzichtete darauf, einer Dimension von Beginn an den Vorzug vor anderen zu geben, obschon insbesondere die deutsche Geschichte eine Dominanz der politisch-rechtlichen Dimension nahelegt.

Der Unterteilung der Gesellschaft entsprechend wird auch der Journalismus in Anlehnung an Weischenberg (2004 [1992]: 71) in die Ebenen Institutionen, Akteure und Aussagen zerlegt, um ihn so empirisch fassbar zu machen. Moderner Journalismus, verstanden als „the sense-making practice of modernity“ (Hartley 1996: 32), wird hierbei definiert über die ihn kennzeichnenden Kerncharakteristika in ihrer historischen Genese und Ausdifferenzierung.

Dabei steht auf institutioneller Ebene die Entwicklung der in Ressorts gegliederten Redaktion als Ort des modernen Journalismus (vgl. Bösch 2005) auf der Basis einer eigenen Presseökonomie (vgl. Stöber 2005a: 19, 2008: 102ff.) in journalistischen Medien (vgl. Weischenberg et al. 2006: 31) im Vordergrund. Innerhalb dieser Strukturen vollzog sich dann die Ausdifferenzierung journalistischer Leitungsrollen (vgl. Blöbaum 1994: 289) auf der Akteursebene. Hier entstanden - im Zuge einer „informellen“ (Requate 2008: 354, 1995: 238) Professionalisierung - ein kohärentes Selbstbild (Kutsch 2008) sowie normierte Arbeitsroutinen von Redakteuren als „journalistisches Handeln“ (Blöbaum 2006: 119, 1994: 136ff.; vgl. Baum 1994). Diese Routinen umfassten die Ausdifferenzierung der Redaktion der journalistischen Inhalte bezüglich Rubrizierung, Aufmachung, Überschriften und Nachrichtenaufbau (vgl. Pöttker 2003, 2005a, 2005b; Wilke 1984, 2001; Weischenberg/Birkner 2008) und zeigten insgesamt die neue gesellschaftliche Aufgabe des Journalismus an, auszuwählen und zu erklären, was das Wichtigste ist.

Insofern geht es hier um die Entstehung des redaktionellen Journalismus nach Baumert (1928: 47ff.) in dreifacher Hinsicht: die Redaktion als Ort, als Akteursgruppe und als Arbeitsweise des Journalismus. Mit der Integration von Sozialgeschichtsschreibung und Kommunikationswissenschaft werden komplementär zu Baumerts eher akteursorientiertem Vorgehen die gesellschaftlichen Rahmenbedingungen in ihrer Bedeutung betont und an den Journalismus rückgebunden. Mit dieser Journalismusgeschichte als Gesellschaftsgeschichte lässt sich eine neue Phaseneinteilung von Genese, Formierung, Ausdifferenzierung und schließlich Durchbruch des modernen Journalismus vornehmen. 


\section{Genese (1605-1848)}

Die Geschichte des Journalismus beginnt 1605 in Straßburg, wo der Nachrichtenhändler Johann Carolus, nachdem er im Jahr zuvor eine Buchdruckerei gekauft hatte, die beiden Gewerbe verband (vgl. Weber 2005; Welke 2008). Doch folgt man den beschriebenen Kerncharakteristika, dann war Carolus erste Zeitung der Welt, Relation, noch kein journalistisches Medium. Die ersten Zeitungen erfüllten, trotz der bereits angelegten Wirtschaftlichkeit (vgl. Birkner 2010: 44), noch nicht das Charakteristikum einer eigenen Presseökonomie, waren sie doch im Buchdruckwesen entstanden und sollten den dort herrschenden Logiken noch über Jahrhunderte gehorchen. Carolus war Drucker und kein Journalist; ein entsprechendes Berufsbild konnte unter den damaligen Bedingungen auch gar nicht entstehen. Weber (2005: 6) kann nicht den „Hauch einer journalistischen Idee" entdecken. Grundsätzlich wurden im 17. Jahrhundert auf der Aussagenebene die Nachrichten zumeist in der Reihenfolge ihres Eingangs aneinandergereiht; Hervorhebungen der Relevanz durch Platzierung, Aufmachung, Überschriften oder einen hierarchischen Nachrichtenaufbau fanden nicht statt (vgl. Wilke 1984: 35ff.).

Damals lebten im Heiligen Römischen Reich Deutscher Nation etwa 20 Millionen Menschen (vgl. Rabe 1991: 617), von denen jedoch erst ein Bruchteil lesen konnte. Der Dreißigjährige Krieg dezimierte einerseits den potenziellen Leserkreis, erhöhte jedoch andererseits massiv die Nachfrage nach überlebenswichtigen Informationen. Deshalb dürften die Nachrichten in den gut einhundert Exemplaren (vgl. Welke 2008: 113; Weber 2005: 14), die Carolus' Relation in Spitzenzeiten als Auflage verzeichnen konnte, auch weit mehr Menschen bekannt geworden sein. Es wurde damals viel vorgelesen.

Die Phase der Genese reichte bis ins 19. Jahrhundert hinein. Nach einer Studie von Jürgen Wilke (1984: 119) lagen Ende des 18. Jahrhunderts bei gut 60 Prozent der Nachrichten ein bis zwei Wochen zwischen dem Datum der Zeitungsausgabe und dem der Nachricht. Der spröde Nachrichtenstil glich noch immer jenem der „Geschriebenen Zeitungen“, die es schon vor Carolus' Relation gegeben hatte (vgl. Weber 2005: 12). Doch wurde das zeitgenössische Medienensemble bereits in dieser Phase durch eine neue Zeitungsform bereichert, die ihre Wurzeln bei den sog. „Moralischen Wochenzeitungen“ hatte und die mit dezidiert politischem und aufklärerischem Anspruch antraten. Hier fand als „Journalismus der schriftstellernden Privatleute“ (Habermas 1990 [1962]: 284) der Bürgerjournalismus vor dem Strukturwandel der Öffentlichkeit statt.

Journalistische Avantgarde waren in der ersten Hälfte des 19. Jahrhunderts vor allem Cottas Allgemeine Zeitung und ihr Pariskorrespondent Heinrich Heine; seine Texte aus Paris sind zeitlose Frühformen der modernen Reportage (vgl. Pöttker 2000, 2008; Haller 2006). Dennoch war Heine ein Schriftsteller, der sich gelegentlich über „dergleichen Geschreibsel“, das „nur für das augenblicklichste Bedürfnis geschrieben“ (2005, 1. Band: 817f.) sei, ärgerte. Ein journalistisches Selbstverständnis konnte sich aber auch deshalb nicht entwickeln, weil „der genuine Journalist“ (Pöttker 2008: 66; Hervorhebung im Original) Heine kaum Kollegen hatte, mit denen er sich über den Journalistenberuf hätte austauschen können. Karl Marx, der im Rheinland wortgewaltig Zeitung machte und durchaus etwas mit Heine zu tun hatte, sprach ebenfalls von sich und seinesgleichen als Schriftsteller (vgl. u. a. Marx/Engels 1969: 19, 1975: 98).

An diesen beiden Aushängeschildern deutscher Intellektualität und Freiheitsliebe wird jedoch exemplarisch besonders deutlich, weshalb es zwar zur Genese, nicht jedoch zur wirklichen Ausdifferenzierung von modernen Charakteristika des Journalismus bis 1848 kommen konnte. Retardierend wirkte in der Dimension von Politik und Recht das Unterdrückungssystem der Karlsbader Beschlüsse von 1819, welches eine „fast dreiBigjährige[...] Polizeiaktion gegen die Presse“ (Schneider 1966: 247) darstellte. Dennoch 
war die Presse wesentlicher Bestandteil der Revolution von 1848, und der Wunsch nach Pressefreiheit blieb auch über die Niederlage 1849 hinaus lebendig (vgl. Wehler 1987b: 779).

\section{Formierung (1849-1873)}

Die faktische Dominanz der Politik in einer „zensurfreien Presseunfreiheit“ (Kohnen 1995: 186) prolongierte sich über die Wendejahre 1848/49 und verschleppte so zunächst eine breite Durchsetzung des redaktionellen Journalismus, obschon sich in der Phase der Genese bei Ausnahmen wie Cottas Allgemeiner Zeitung und der Kölnischen Zeitung bis zur Mitte des 19. Jahrhunderts auf institutioneller Ebene redaktionelle Strukturen herausgebildet hatten (Requate 1995: 129, 214; vgl. Heyck 1898: 155; Buchheim 1930: 44ff., 1976: 98ff.). Die Zeitung als Wirtschaftsprodukt litt nach 1848/49 unter politischen und wirtschaftlichen Einschränkungen, namentlich den drei „C“ - Conzession, Caution, Confiskation (vgl. Verlag der Frankfurter Zeitung 1911: 385). Eben weil der gesamtgesellschaftliche Rahmen noch nicht gespannt war, konnte die „Periode des redaktionellen Journalismus“ (Baumert 1928: 47) nicht schon 1848 beginnen.

Dennoch stellen sich in der Zusammenschau von endogenen und exogenen Faktoren die Jahre 1848/49 als erste große Zäsur in der Geschichte des Journalismus heraus; mit der Revolution, ihrer Niederlage und den Auswirkungen von beidem auf den deutschen Journalismus. Wichtigstes Element der anschließenden Formierungsphase war die symbiotische Entstehung von Parteiungen und Parteizeitungen, weshalb die Forschung hier von der „Epoche der politischen Parteipresse“ (Koszyk 1966: 128) spricht. Prägend für diese Zeit waren aber auch Entwicklungen in der Dimension von Wirtschaft und Technologie.

Die Erfindung der Telegraphie revolutionierte die öffentliche Kommunikation. Eric Hobsbawm (1980: 80) meint gar: „Vom journalistischen Standpunkt aus gesehen endete das Mittelalter in den sechziger Jahren des 19. Jahrhunderts, als es möglich wurde, Nachrichten aus aller Welt ungehindert von so vielen verschiedenen Orten des Globus aus zu kabeln, dass sie rechtzeitig am nächsten Morgen auf dem Frühstückstisch lagen. “ Und während in der Kölnischen Zeitung am 5. Oktober 1849 „das erste ,elektrische’ Telegramm“ in Fettdruck auf der ersten Seite gestanden haben soll (Weinhold 1969: 145), klagte der Chefredakteur der Allgemeinen Zeitung, Gustav Kolb, im Jahr darauf seinem Verleger Cotta, man werde von den großen Blättern aus „Leipzig, Hannover, Bremen, Berlin, Wien, Köln, Hamburg überflügelt, welche täglich telegr. Berichte haben, und zwar oft zehn bis zwölf am Tage. "1 Die Allgemeine Zeitung verschlief diese journalistische Weiterentwicklung, nahm nicht Teil am neuen Race for News, ihre Auflagenzahlen sanken (vgl. Müchler 1998: 196; Fischer 2005: 25ff.; Requate 1995: 283), und so löste die wirtschaftlich erfolgreichere Kölnische Zeitung (vgl. Weinhold 1969: 145f.; Buchheim 1976: 167ff.) sie als führendes Blatt in deutschen Landen ab. In dieser Phase eines langen Aufschwungs der sich globalisierenden Weltwirtschaft reüssierten jene Zeitungen, welche sich an den neuen Puls der Zeit anschlossen.

Dabei wurde auch das Anzeigengeschäft populärer, hatte es aber zunächst in der politischen Parteipresse in Deutschland enorm schwer. Ferdinand Lassalle (1919 [1863]: 360ff., 1984 [1863]: 229ff.) hatte sich so kategorisch gegen Werbung in Zeitungen ausgesprochen, dass dies für die deutsche Sozialdemokratie zu einer Art Dogma wurde (vgl. Wolter 1981: 161). Erst 1892 erklärte August Bebel, es sei „eine Torheit, auf Überschüsse

1 Aktenbestand im Cotta Archiv (Stiftung Stuttgarter Zeitung), Briefe, Kolb an Cotta, 17. September 1850, Cotta Br. Kolb No. 908. Hervorhebung im Original. 
zu verzichten, die durch Inserate erzielt werden könnten“ (zit. n. Koszyk 1966: 203, vgl. ebd.: 269; Retallack 1993: 202; Brunhuber 1908: 98).

Während der Formierungsphase wurde vor allem das Fundament für die noch gehemmten Weiterentwicklungen gelegt. Das deutsche Bildungssystem brachte zunehmend die Produzenten wie auch die Konsumenten journalistischer Produkte hervor. Die Gymnasien bildeten für den Journalismus besonders geeignete „Generalisten“ (Wehler 1995: 412) aus. Die Frauen, denen der Zugang zu höherer Bildung verwehrt blieb, spielten vor allem als Konsumentinnen familientauglicher journalistischer Produkte eine entscheidende Rolle (vgl. Wischermann 1983, 1996). Dennoch bedurfte es einer weiteren Zäsur Anfang der 1870er Jahre, ehe die Formierungsphase der modernen Gesellschaft in die Formulierungsphase des gesellschaftlichen Selbstgesprächs eintreten sollte.

\section{Ausdifferenzierung (1874-1900)}

In Folge der Reichsgründung 1871 traten nun im Rahmen des deutschen Nationalstaats in allen Dimensionen der hier wissenschaftlich zerlegten Gesellschaft die entscheidenden Veränderungen ein. War bereits in der Formierungsphase die Bevölkerung von 33,7 Millionen Menschen 1850 auf über 41 Millionen im Jahr 1871 angewachsen, so lebten schließlich 1910 knapp 65 Millionen im Deutschen Reich (vgl. Wehler 1995: 9, 512). Entscheidend für den Journalismus aber war nicht so sehr das Bevölkerungswachstum an sich, sondern das Zusammenspiel der gesamtgesellschaftlichen Großtrends von Urbanisierung und Alphabetisierung (Engelsing 1973: 98): „1871 wurde anhand verschiedener Kriterien die Zahl der Analphabeten in Preußen im Alter über zehn Jahre noch mit 10 Prozent der Männer und 15 Prozent der Frauen, zusammen mit 12 Prozent, 1899 mit je 1 Prozent der Männer und Frauen unter den Eheschließenden festgestellt." $\mathrm{Zu}$ Beginn des Kaiserreichs hatten noch 60 Prozent der Bevölkerung auf dem Land gelebt, 1910 lebten bereits gut 60 Prozent in Städten (vgl. Wehler 1995: 512).

Das neue menschliche Miteinander in der Großstadt, dessen Zusammenhänge nur noch medial vermittelt werden konnten, und die neuen technischen Möglichkeiten von immer mehr und immer schnelleren Nachrichten setzten schließlich flüchtigere Lesegewohnheiten durch (vgl. u. a. Löbl 1903: 115; Pöttker 2005b: 21). Das Reichspressegesetz von 1874 (vgl. u. a. Stöber 2005b: 146ff.; Naujoks 1975; Wetzel 1975; Wehle 1883: 150ff.) bot einen rechtlich nicht besonders liberalen, aber doch stabilen Rahmen, der insbesondere die wirtschaftlichen Fesseln der Presse erheblich lockerte und damit geradezu einen Boom auslöste. Insbesondere fand der gesellschaftlich notwendig gewordene Ausbau der Redaktionen auf der neuen Basis der Anzeigenfinanzierung statt, wobei darauf geachtet wurde, dass „einer, der neue Matjesheringe anzeigt, deshalb nicht die Haltung der Zeitung in der Flottenfrage bestimmen" könne, so Julius Bachem (1906: 18) von der Kölnischen Volkszeitung.

Die Ökonomisierung der Medien trat nun in die dritte und entscheidende Phase (vgl. Birkner 2010: 46), und die in diesem Zusammenhang neu auftretende Generalanzeigerpresse verkörperte zunächst den starken Einfluss des Wirtschaftssystems (vgl. Engelsing 1966: 271). Aber zwischen der vom Gesinnungsjournalismus gepflegten Parteilichkeit und der unterstellten Parteilosigkeit der Generalanzeiger wurde langsam, aber sicher eine Un- bzw. Überparteilichkeit möglich. „Man sehe sich einmal“, so der zeitgenössische Beobachter Robert Brunhuber (1908: 97f.), „nach einer großen Redeschlacht im Reichstag die zehn größten Organe der zehn wichtigsten Parteien durch: In jedem Organ hat mit unzweifelhafter Sicherheit der Redner der betreffenden Partei den Vogel abgeschossen, die andern Gegner liegen im Staub der politischen Arena.“ Er kritisierte eine platte Parteilichkeit, welche nicht mehr den Bedürfnissen einer Demokratie entspräche. Da- 
gegen führte er eine Reihe demokratischer Blätter an, die sich dezidiert gegen eine Parteizugehörigkeit aussprachen. So erklärte zum Beispiel das Berliner Tageblatt, es könne nicht „die Aufgabe eines liberalen Blattes sein, mit einer bestimmten Richtung durch dick und dünn zu gehen“ (ebd.: 98).

Frank Bösch (2006: 120) spricht von einem Transformationsprozess des deutschen Journalismus ab den 1880er Jahren, vor allem begünstigt durch die „Ausdehnung des Wahlrechts und die Ausbildung der Massenpresse“. Spätestens seit dem Ende der Sozialistengesetze 1888 ebenso wie nach dem Ende der Ära Bismarck zwei Jahre später kann für das Deutsche Kaiserreich in Friedenszeiten mit einiger Berechtigung von einer faktischen - jedoch stets fragilen - Pressefreiheit gesprochen werden. Das führte dazu, dass die Politik zunehmend dem Einfluss der Medien ausgesetzt war und sich über die Medien auch dem Urteil der Öffentlichkeit stellen musste. Nun war die gesellschaftliche Beobachtung durch Journalismus eben nicht mehr nur nötig, sondern auch möglich.

\section{Durchbruch (1900-1914)}

Für eine Phaseneinteilung sind Zäsuren zwingend notwendig, obwohl sie häufig den evolutionären Charakter von sich langsam vollziehenden Wandlungsprozessen unterschlagen. Das gilt auch für die Zäsur um 1900. Kaspar Maase (1997: 20; vgl. Faulstich 2002: 203f.) gibt zu, dass diese Zäsur „wie jede andere, ein Moment von Willkür“ habe, und dennoch setzt er „die endgültige Etablierung der eigentlich modernen Massenkultur im Jahrzehnt vor dem Ersten Weltkrieg “ an. Der Journalismus war integraler Bestandteil der „Entfesselung der Massenkommunikation“ (Wilke 2000: u. a. 115, 275, 287) und konnte sich in den Jahren von 1900 bis 1914 entsprechend als System der gesellschaftlichen Selbstbeobachtung durchsetzen. Im Sinne einer weiteren Ausdifferenzierung vollzog sich nun auf allen drei bereits generierten, formierten und ausdifferenzierten Ebenen der journalistischen Institutionen, Akteure und Aussagen der Durchbruch des modernen Journalismus.

Um dies nachweisen zu können, wird hier auf eine bei der Rekonstruktion der deutschen Journalismusgeschichte bislang wenig systematisch analysierte Textgattung zurückgegriffen: die Praktikerliteratur (vgl. aber Groth 1948, Requate 1995). Otto Groth erklärt den Entstehungszusammenhang der von ihm sog. Praktikerliteratur (Groth 1948: 301) mit der Selbstfindung des Berufs (ebd.: 302): „Der Journalismus, in den während der sechziger und siebziger Jahre die heterogensten Elemente eingeströmt waren, konsolidierte sich allmählich im Geistlichen wie Organisatorischen und strebte eine Hebung des intellektuellen und ethischen Niveaus, eine Sicherung der wirtschaftlichen Stellung und eine Besserung des sozialen Ansehens an. " Eine solche Kontextualisierung der Texte ist für das geschichtswissenschaftliche Quellenstudium als historische Methode ebenso wichtig wie für die qualitative Inhaltsanalyse (Borowsky/Vogel/Wunder 1989: 157ff.; Mayring 2008: 42ff., insb. 46f.).

Die ersten Jahre des 20. Jahrhunderts stellten in ganz Europa (vgl. Blom 2009), insbesondere aber in Deutschland, eine Zeit rasanter gesellschaftlicher Modernisierung dar. Vor diesem sozio-kulturellen Hintergrund zwang die sich immer stärker arbeitsteilig organisierende Gesellschaft auch das entstehende journalistische System zu einer stärkeren Selbstverständigung über Normen und Regeln, wie sie dann in der Praktikerliteratur in diesem Zeitraum niedergeschrieben wurden. Damit richteten sich die Autoren sowohl an ihre eigene Berufsgruppe als auch an die Gesellschaft insgesamt. 
Tabelle 1: Auswabl journalistischer Praktikerliteratur zu Beginn des 20. Jabrbunderts

\begin{tabular}{lll}
\hline Autor & Titel & Erscheinungsjahr \\
\hline Johannes Frizenschaf & Die Praxis des Journalisten & $(1901)$ \\
Richard Jacobi & Der Journalist & $(1902)$ \\
Richard Wrede & Handbuch der Journalistik & $(1906)[1902]$ \\
Emil Löbl & Kultur und Presse & $(1903)$ \\
Hermann Diez & Das Zeitungswesen & $(1910)$ \\
Friedrich Streißler & Schriftsteller und Journalist & $(1912)$ \\
Albert Haas & Das moderne Zeitungswesen & $(1914)$ \\
\hline
\end{tabular}

Ein Teil dieser Bücher, wie Kultur und Presse von Emil Löbl, Die Zeitung von Jacob Julius David und Das moderne Zeitungswesen von Albert Haas, waren eher zeitgenössische wissenschaftliche Reflexionen über den Zusammenhang von Journalismus und Gesellschaft. Dagegen waren Johannes Frizenschafs Die Praxis des Journalisten, Richard Jacobis Der Journalist, Richard Wredes Handbuch der Journalistik und Friedrich Streißlers Schriftsteller und Journalist auf die journalistische Praxis orientiert. Die Analyse dieser Texte offenbart ein größtenteils übereinstimmendes Bild über journalistische Institutionen, Akteure und Aussagen nach der Jahrhundertwende.

\subsection{Moderne journalistische Institutionen}

Das Pressewesen boomte in den ersten Jahren des 20. Jahrhunderts. Die Zahl der Zeitschriften stieg von 5.632 (1902) auf 6.689 (1913) an (Wilke 2000: 277; vgl. Stöber 2005b: 266ff.). Auch die Zahl der Zeitungen wuchs von 3.405 (1897) auf 4.221 Zeitungen 1914 (vgl. Wilke 2000: 259ff.). Die Gesamtauflage der deutschen Zeitungen kann zu jener Zeit auf 18 bis sogar 25 Millionen geschätzt werden (vgl. Stöber 2005b: 161). Diese Massen waren unter den Bedingungen des Buchdrucks längst nicht mehr zu bewältigen. Der moderne Zeitungsdruck fand nun auf eigenen Rotationsmaschinen und unter massivem Einsatz von Ressourcen statt. Für die Herstellung des Hamburger Fremdenblatts vom 14. Dezember 1913 waren knapp 35000 Kilogramm Papier erforderlich, das entspricht 3 1/2 Eisenbahn-Doppelwaggons (vgl. Bertheau 1914: 90).

Auf der Basis einer eigenständigen Presseökonomie, bei der die Redaktion im Verlagshaus Teil der Fixkosten ist - sozusagen das Gegenteil von „Outsourcing“ -, vollzog sich die Ausdifferenzierung der Redaktionen in Ressorts (vgl. Stöber 2008: 102ff.). Die Redaktion des Berliner Lokal-Anzeigers war von drei Redakteuren 1883 auf 34 (vgl. Requate 1995: 154), bzw. 46 Redakteure (vgl. Stöber 1994: 324; Meier 2002: 125, 2005 : 395; Dussel 2004: 92; Bösch 2005: 73) im Jahre 1899 angewachsen und war damit wohl eine der größten Redaktionen in Deutschland.

Die moderne Zeitungsredaktion, deren Genese 1605 in Straßburg begann, hatte nun „zahlreiche Charakteristika ausgebildet, die trotz aller technischen Innovationen bis heute ihre materielle Struktur, ihre soziale Praxis und ihre Repräsentation prägen“ (Bösch 2005: 76). Jürgen Wilke (2002, 2003) hat gezeigt, dass man anhand von Bauplänen von Verlagsgebäuden die Ressortstruktur der Redaktionen nachweisen kann. Die organisatorischen Veränderungen verschmolzen nun dahin, dass die Redaktion den Zeitungsinhalt thematisch gruppierte und umgekehrt.

Das lässt sich auch mit der Analyse der vorgestellten Praktikerliteratur nachweisen. Einzig Löbl (1903: 44ff.) unterteilte die Zeitung eher philosophisch in einen referierenden, einen räsonierenden und einen schöngeistigen Teil und beschrieb hiermit im Kern die Dreiteilung in Nachrichten-, Meinungs- und Unterhaltungs-Darstellungsformen 
(vgl. Weischenberg 2001: 49ff.). Die weiteren Autoren benannten sämtlich die „klassischen" Ressorts von Politik, Feuilleton, Wirtschaft/Handelsteil und Lokales und zwar im Kontext von „Zeitungsrubriken“ (Frizenschaf 1901: 82f.), „redaktionellen Ressorts“ (Jacobi 1902: 113, 117ff.), „Ressortredakteure“ (Wrede 1906 [1902]: 116f.), „Sparten“(Diez 1910: 84), „Teile des Blattes“ (Streißler 1912: 16f.) und „Ressorts“ (Haas 1914: 12). In den jüngeren Werken von Diez, Streißler und Haas fand außerdem (jeweils ebd.) das jüngste Ressort, der Sportteil, Erwähnung. Dessen Genese und Ausdifferenzierung lässt sich seit den 1870er Jahren in Deutschland nachweisen, der richtige Durchbruch erfolgte jedoch auch hier erst nach 1900 (vgl. u. a. Weischenberg 1978 [1976]: 126ff.; Blöbaum 1994: 210; Stöber 2005b: 214). Innerhalb der ausdifferenzierten Ressortstrukturen vollzog sich nun auch die Ausdifferenzierung von journalistischen Leistungsrollen (vgl. Blöbaum 1994: 289). Streißler (1912: 17) beschrieb sehr detailliert die Binnendifferenzierungen des Berufs:

„Da ist die Arbeitsteilung soweit fortgeschritten, daß für die verschiedenen Teile des Blattes besondere Redakteure angestellt sind. Der Chefredakteur hat da in der Hauptsache nur dafür zu sorgen, daß die einzelnen Kräfte richtig zusammen arbeiten. Da ist ein Chefredakteur für den lokalen Teil, ein Redakteur für den Sport, für dessen verschiedene Betätigungen (Rennsport, Radfahrsport, Wassersport, Luftschiffahrt usw.) noch besondere Mitarbeiter und Berichterstatter vorhanden sind. Das Feuilleton hat seinen besonderen Redakteur, der wieder besondere Hilfskräfte für Theater, Musik und Literatur hat. Auch die Wissenschaft untersteht einem Redakteur, und die Politik ist meist unter zwei Kräften verteilt, nämlich eine für die innere und eine für die äußere Politik. Ebenso hat der Handelsteil seinen besonderen Redakteur mit mehreren Unterredakteuren, von denen jeder sein bestimmtes Gebiet bearbeitet.“

\subsection{Moderne journalistische Akteure}

Paul Stoklossa hat 1910 den Arbeitsmarkt der Redakteure untersucht und dabei eine auch einkommensdeterminierende Rangfolge aufgestellt (1910: 532):

- Chefredakteur, Hauptredakteur,

- Politischer Redakteur, erster Redakteur, Handels-, Feuilleton-, Ressortredakteur,

- Lokalredakteur,

- Hilfsredakteur, zweiter Redakteur, Redaktionsgehilfe, Volontär.

Eher am unteren Ende dieser Liste oder gar hauptsächlich frei arbeitend fand sich die kleine Zahl der Journalistinnen des Kaiserreichs. Elisa Ichenhaeuser hat die Journalistik als Franenberuf untersucht und festgestellt, dass sich das Gros der Journalistinnen mit „einer jämmerlichen Bezahlung begnügen“ (1905: 13) musste. Stoklossas Untersuchung zeigt, wie sehr sich mittlerweile in den Redaktionen horizontal wie vertikal Leistungsrollen ausdifferenzierten. Die Etablierung des Redakteursberufs machte auch eine professionelle Abgrenzung von anderen Berufen notwendig.

James Retallack (1993: 205) sieht die Praktikerliteratur im Zusammenhang eines sich findenden professionellen (Selbst)bewusstseins im Journalismus: „[T]he sheer number of handbooks promising ,practical tips' and ,professional advice' to journalists after the 1880 s - as well as their growing size, their frequent reprintings, and their authors' effort to engage each other in debate - attests to the fact that a new level of ,professional' (self-) consciousness had been reached. " Hier wurden für Kollegen, freie Mitarbeiter und auch angehende Redakteure die Funktionen und die Normen eines entstehenden Berufes niedergeschrieben.

Die stärker praxisorientierten Autoren Frizenschaf (1901: 102f.) und Jacobi (1902: 134f.) zitierten zum Beispiel die „Preßgebote“ der Kölnischen Volkszeitung: „Dein Grundsatz sei: Thatsachen, keine Phrasen, keine langen Betrachtungen“; „Eigennamen, 
besonders Ortsnamen, die im Text vorkommen, schreibe am Rande nochmals und zwar mit lateinischen Buchstaben“; „Mache keine langen Sätze“. Diese Hinweise waren zunächst dezidiert an Personen außerhalb der Redaktionen gerichtet, an freiberufliche Journalisten und an die zahlreichen Leser, die den Zeitungen Beiträge einsandten. Es wurden die Regeln für den Systemeintritt ebenso formuliert wie jene innerhalb des Systems. Löbl ermahnte die Redakteure, sie sollten klar und leicht verständlich schreiben (1903: 120), nicht pöbeln (ebd.: 129) und vorsichtig mit dem Wort „bekanntlich“ (ebd. 138) umgehen:

„Welcher Missbrauch wird ferner mit dem ,bekanntlich’ getrieben! ,Bekanntlich’ sind von den 67 Orkneyinseln nur 28 bewohnt, ,bekanntlich' ist Erasmus v. Patow am 10. September 1804 geboren, ,bekanntlich' wird Natriumnitrit zur Darstellung der Diazoverbindungen benützt. Was einer vor fünf Minuten aus dem Konversationslexikon erfahren hat, stellt er als bekannte Tatsache hin, um seine Polyhistorie in das rechte Licht zu setzen."

Jacobi und Wrede definierten den Journalisten als einen, der „Tag um Tag in einer Zeitung veröffentlicht“ (Jacobi 1902: 100) bzw. der „für Zeitungen und Zeitschriften gewerbsmäßig gegen Bezahlung“ (Wrede 1906 [1902]: 3) arbeitet. Über die tagtäglichen Aufgaben herrschte mittlerweile weitgehender Konsens in der Zunft. Fritzenschaf (1901: 85) nannte das Unterkapitel entsprechend: „Beschaffung, Auswahl und Behandlung des Zeitungsstoffes“. Während die Autoren Wrede (1906 [1902]: 119f.), Löbl (1903: 54ff.), Streißler (1912: 14f.) und Haas (1914: 6) die journalistischen Kerntätigkeiten eher zusammenhanglos aufzählten, waren sie bei Diez (1910: 90) als Herbeischaffung, Sichtung, Ergänzung, Anordnung und schließlich Redigieren expliziert. Am präzisesten beschrieb Jacobi (1902: 105) die „Tätigkeit des Redakteurs“: „Lesen, Redigieren, Schreiben, Korrigieren, Informieren“. Die charakteristischen Arbeitsroutinen von „Recherchieren, Selektieren, Schreiben und Redigieren“ (Malik 2004: 89) hatten sich um 1900 ausdifferenziert. Vor allem die Auswahl wurde immer wieder thematisiert; sie war durch die $\mathrm{Zu}-$ nahme des Nachrichtenstoffs enorm wichtig geworden. Jetzt erst wurde der Journalist zum „Gatekeeper“ für den Rezipienten. Das bestätigt sich auch bei Fritzenschaf (1901: 61) und Haas (1914: 16, 29), die die vornehmliche Funktion des Journalisten darin sahen, das Wichtige vom Unwichtigen zu scheiden.

\subsection{Moderne journalistische Aussagen}

Zunehmend wurden die Nachrichten durch die Anordnung ihrer Bedeutung und dem Publikumsinteresse entsprechend gewertet. Der General-Anzeiger für Hamburg-Altona schrieb am 22. Oktober 1893 nicht ohne Selbstbewusstsein, dass „ein Blick auf die erste Seite eines General-Anzeigers genügt, um die wichtigste Tagesneuigkeit zu erfahren" (zit. nach Wolter 1981: 349). Aufmachung und Rubrizierung des Nachrichtenstoffs setzten sich durch.

Überschriften sorgten nun für eine neue Übersichtlichkeit als Nachricht über der Nachricht (vgl. Schneider/Esslinger 2007: 11). Und auch der Aufbau des Nachrichtentextes folgte nun stärker dem im amerikanischen Journalismus zunächst herausgebildeten Pyramidenschema (vgl. Weischenberg 2001: 79ff.; Wilke 2001; Pöttker 2003, 2005a, 2005b; Weischenberg/Birkner 2008), welches die Meldung hierarchisch nach der unterstellten Wichtigkeit der Fakten sortiert. In der Frankfurter Zeitung (zweites Morgenblatt) vom 26. August 1906 war auf Seite zwei zum Beispiel unter der Überschrift „Zum Bierkrieg“"zu lesen:

„Duisburg, 24. August. Der hiesige Wirteverein beschloss einstimmig, die von den Brauereien geforderte Bierpreiserhöhung nicht zu zahlen. Es wurde eine Kommission gewählt, die die Verträge 
der abhängigen Wirte prüfen und dann mit den Brauereien unter Zuziehung eines Rechtsanwalts in Verhandlungen treten soll. In der Versammlung wurde bekannt gegeben, daß die Brauereien auch den Preis für Flaschenbier erhöhen wollen. Vom 1. Januar 1907 soll die 4-Zehntel-Literflasche 8 Pfg. und vom 1. April 1907 ab 10 Pfg. kosten.“

Diese moderne Nachricht folgt einem hierarchischen Aufbau. Die wichtigsten W-Fragen Wer, Wie, Was werden gleich im ersten Satz beantwortet und entsprechend ihrer Relevanz weitere Detailinformationen angehängt.

Teil der journalistischen Modernisierung waren auch sich ausdifferenzierende Textstrukturen. In der Praktikerliteratur wurde ein weit aufgefächertes Repertoire an Darstellungsformen, verstanden als „Chiffre für ein erwartbares kommunikatives Geschehen" (Pörksen 2006: 193) zwischen Produzenten und Rezipienten, thematisiert. Einzig Streißler, der sich mit seinem Buch vor allem an freie Schriftsteller wandte, erwähnte nur das Feuilleton (1912: 41). In den anderen Werken wurden zumeist Leitartikel, Nachrichten, Lokal- oder Parlamentsbericht, Korrespondenzen und Feuilleton genannt (Frizenschaf 1901: 83; Jacobi 1902: 117f.; Wrede 1906 [1902]: 130f.; Diez 1910: 84f.; Streißler 1912: 16f.; Haas 1914: 16f), bei Löbl (1903: 99) war dies dann eher im Sinne einer „Rubrikeneinteilung“ die „äußere Einteilung des Blattes“. Einige erwähnten darüber hinaus noch die Reportage (Löbl 1903: 182; Diez 1910: 85; Haas 1914: 167) und das Interview (Löbl 1903: 54; Haas 1914: 16), allerdings mehr als Recherchetechnik denn als Darstellungsform. Das Interview hatte es unter den Bedingungen der politischen Gängelung in Deutschland nicht leicht gehabt und gewann doch um 1900 zunehmend an Bedeutung (vgl. Grzella/Pfingsten 1994; Bösch 2009: 408ff.). Es zeigt ebenso wie die Nachrichtenpyramide die neue Rolle der Journalisten in der Gesellschaft an (Schudson 1991: 438): "The summary lead, much as we may take it for granted today, was a literary invention that asserted the journalist's authority to define for readers the most important element of a news event."

\section{Fazit}

Erst die deutsche Kaiserreichsgesellschaft hatte bis 1900 ein modernes Selbstbeobachtungssystem ausdifferenzieren können, welches sich dann nach 1900 vollends entfalten konnte. Bis dahin hatte vor allem die Dominanz der politisch-rechtlichen Dimension zwar nicht so sehr die Genese, sehr wohl aber die Ausdifferenzierung des Journalismus behindert, verlangsamt und verkompliziert. So folgte auf die sehr lange Phase der Genese von 1605 bis 1848 zunächst eine Formierungsphase in den Jahren 1849 bis 1873, in welcher die im Journalismus bereits angelegten Strukturen zunächst verfestigt wurden. Erst im Nationalstaat kamen die modernen Trends der Hochindustrialisierung und der Urbanisierung dann voll zum Tragen, veränderten die gesellschaftliche Kommunikation und beförderten zugleich Fragmentierung und Vermassung. Jetzt differenzierten sich die Leserschaft und das Stoffangebot geradezu universell aus.

Um seiner Funktion der gesellschaftlichen Selbstbeobachtung nachkommen zu können, hatte der Journalismus in der Folge Einrichtungen, Einsichten und Praktiken der Sinnherstellung entwickelt. Dies geschah in einem langsamen evolutionären Prozess, in welchem sich schließlich in der Phase der Ausdifferenzierung nach dem Reichspressegesetz 1874 bis 1900 auf institutioneller Ebene Ressortstrukturen, auf der Akteursebene journalistische Leistungsrollen und auf der Ebene der Aussagenproduktion journalistische Arbeitsweisen ausdifferenzierten. In den ersten Jahren des 20. Jahrhunderts brach dann der moderne Journalismus auf allen bereits angelegten Ebenen durch, wie sich mit der zeitgenössischen Praktikerliteratur nachweisen lässt. 
Die Modernität des deutschen Journalismus zu Beginn des 20. Jahrhunderts ist bislang unterschätzt worden. Zu dominant erschienen die Zwänge der politisch-rechtlichen Dimension in den vorherigen Epochen und zu glatt ließen sich diese mit der Zensur im Ersten Weltkrieg, der Polarisierung in der Weimarer Republik und der Totalität im Nationalsozialismus zu einer unendlichen Geschichte eines vormodernen Journalismus in Deutschland verknüpfen. Doch auch in Deutschland begann um 1900 das „Jahrhundert des Journalismus“ (Birkner 2010).

\section{Literatur}

Bachem, Julius (1906): Allerlei Gedanken über Journalistik. Leipzig: Duncker \& Humblot.

Baum, Achim (1994): Journalistisches Handeln. Eine Kritik der Journalismusforschung, Opladen: Westdeutscher Verlag.

Baumert, Dieter Paul (1928): Die Entstehung des deutschen Journalismus. Eine sozialgeschichtliche Studie. München, Leipzig: Duncker \& Humblot.

Bertheau, Franz R. (1914): Kleine Chronologie zur Geschichte des Zeitungswesens in Hamburg von 1616 bis 1913. Hamburg: Realschule vor dem Lübeckertore.

Birkner, Thomas (2010): Das Jahrhundert des Journalismus. Ökonomische Grundlagen und Bedrohungen. In: Publizistik 55, Nr. 1, 41-54.

Blöbaum, Bernd (1994): Journalismus als soziales System. Opladen: Westdeutscher Verlag.

Blöbaum, Bernd (2006): Journalistisches Handeln. In: Günter Bentele, Hans-Bernd Brosius, Otfried Jarren (Hrsg.), Lexikon Kommunikations- und Medienwissenschaft, Wiesbaden: VS, 119.

Blom, Philipp (2009): Der taumelnde Kontinent. Europa 1900-1914. München: Hanser.

Borowsky, Peter; Vogel, Barbara; Wunder, Heide (1989): Einführung in die Geschichtswissenschaft I: Grundprobleme, Arbeitsorganisation, Hilfsmittel. 5. Auflage. Opladen: Westdeutscher Verlag.

Bösch, Frank (2005): Die Zeitungsredaktion. In: Alexa Gisthövel, Habbo Knoch (Hrsg.), Orte der Moderne. Erfahrungswelten des 19. und 20. Jahrhunderts, Frankfurt/New York: Campus, 71-80.

Bösch, Frank (2006): Volkstribune und Intellektuelle: W. T. Stead, Maximilian Harden und die Transformation des politischen Journalismus in Deutschland und Großbritannien. In: Clemens Zimmermann (Hrsg.), Politischer Journalismus, Öffentlichkeiten und Medien im 19. und 20. Jahrhundert. Ostfildern: Jan Thorbecke Verlag, 99-120.

Bösch, Frank (2009): Öffentliche Geheimnisse. Skandale, Politik und Medien in Deutschland und Großbritannien 1880-1914. München: Oldenburg.

Bourdieu, Pierre (2005): The Political Field, the Social Field, and the Journalistic Field. In: Rodney Benson, Erik Neveu (Ed.), Bourdieu and the Journalistic Field. Cambridge: Polity, 29-47.

Brunhuber, Robert (1908): Das Deutsche Zeitungswesen. Leipzig: G. J. Göschen'sche Buchhandlung.

Buchheim, Karl (1930): Geschichte der Kölnischen Zeitung, ihrer Besitzer und Mitarbeiter, 2. Band: Von den Anfängen Joseph DuMonts bis zum Ausgang der deutschen bürgerlichen Revolution - 1831-1850. Köln: M. DuMont Schauberg.

Buchheim, Karl (1976): Geschichte der Kölnischen Zeitung, ihrer Besitzer und Mitarbeiter, 3. Band: Der Aufstieg zur Weltpresse im Preußen der Reaktion - 1850-1858. Köln: M. DuMont Schauberg.

Diez, Hermann (1910): Das Zeitungswesen. Leipzig: B. G. Teubner.

Dussel, Konrad (2004): Deutsche Tagespresse im 19. und 20. Jahrhundert. Münster: Lit.

Engelsing, Rolf (1966): Massenpublikum und Journalistentum im 19. Jahrhundert in Nordwestdeutschland. Berlin: Duncker \& Humblot.

Engelsing, Rolf (1973): Analphabetentum und Lektüre. Zur Sozialgeschichte des Lesens in Deutschland zwischen feudaler und industrieller Gesellschaft. Stuttgart: J. B. Metzler.

Faulstich, Werner (2002): Einführung in die Medienwissenschaft: Probleme, Methoden, Domänen. München: Fink. 
Fischer, Bernhard (2005): Die Augsburger „Allgemeine Zeitung“ 1798-1866. Nach dem Redaktionsexemplar im Cotta-Archiv (Stiftung der Stuttgarter Zeitung), Register der Beiträger/Mitteiler, Teil 3: 1850-1866. Im Auftrag des Deutschen Literaturarchivs bearbeitet von Bernhard Fischer. München: K.G. Saur.

Frizenschaf, Johannes (1901): Die Praxis des Journalisten. Ein Lehr- und Handbuch für Journalisten, Redakteure und Schriftsteller. Leipzig: Walther Fiedler.

Gebhardt, Hartwig (2007): Mir fehlt eben ein anständiger Beruf. Leben und Arbeiten des Auslandskorrespondenten Hans Tröbst (1891-1939). Bremen: edition lumière.

Groth, Otto (1948): Die Geschichte der deutschen Zeitungswissenschaft. Probleme und Methoden. München: Weinmayer.

Groth, Otto (1962): Die unerkannte Kulturmacht. Grundlegung der Zeitungswissenschaft (Periodik). 4. Band. Berlin: Walter de Gruyter \& Co.

Grzella, Stefanie; Pfingsten, Stefan (1994): Genese einer journalistischen Darstellungsform: Das Interview in Wien und Berlin zwischen 1860 und 1900. Eine empirische Untersuchung anhand ausgewählter Zeitungen. Diplomarbeit am Institut für Journalistik, Universität Dortmund.

Haas, Albert (1914): Das moderne Zeitungswesen in Deutschland. In: Volkswissenschaftliche Gesellschaft in Berlin (Hrsg.), Volkswirtschaftliche Zeitfragen. Vorträge und Abhandlungen. Berlin: Verlag von Leonhard Simion NF, 1-35.

Haas, Hannes (1999): Empirischer Journalismus. Verfahren zur Erkundung gesellschaftlicher Wirklichkeit. Wien, Köln, Weimar: Böhlau.

Habermas, Jürgen (1990 [1962]): Strukturwandel der Öffentlichkeit. Untersuchung zu einer Kategorie der bürgerlichen Gesellschaft. Frankfurt am Main: Suhrkamp.

Haller, Michael (2006): Die Reportage, 5., überarbeitete Auflage. Konstanz: UVK.

Hartley, John (1996): Popular Reality: Journalism, Modernity, Popular Culture. London: Arnold.

Heine, Heinrich (2005): Sämtliche Schriften. Herausgegeben von Klaus Briegleb. 6 Bände. München: dtv.

Heyck, Eduard (1898): Die Allgemeine Zeitung 1798-1898. München: Verlag der Allgemeinen Zeitung.

Hickethier, Knut (2003): Gibt es ein medientechnisches Apriori? Technikdeterminismus und Medienkonfiguration in historischen Prozessen. In: Markus Behmer, Friedrich Krotz, Rudolf Stöber, Carsten Winter (Hrsg.), Medienentwicklung und gesellschaftlicher Wandel. Wiesbaden: Westdeutscher Verlag, 39-52.

Hobsbawm, Eric J. (1980): Die Blütezeit des Kapitals. Eine Kulturgeschichte der Jahre 1848-1875. Frankfurt am Main: Fischer.

Hömberg, Walter (1987): Von Kärrnern und Königen. Zur Geschichte journalistischer Berufe. In: Manfred Bobrowsky, Wolfgang R. Langenbucher (Hrsg.), Wege zur Kommunikationsgeschichte. München: Ölschläger, 619-629.

Ichenhaeuser, Elisa (1905): Die Journalistik als Frauenberuf. Berlin/Leipzig: Verlag der Frauenrundschau Schweizer \& Co.

Jacobi, Richard (1902): Der Journalist. Hannover: Jänecke.

Kinnebrock, Susanne (2005): Frauen und Männer im Journalismus. Eine historische Betrachtung. In: Martina Thiele (Hrsg.), Konkurrenz der Wirklichkeiten. Göttingen: Göttinger Universitätsverlag, S. 101-132.

Kohnen, Richard (1995): Pressepolitik des Deutschen Bundes. Methoden staatlicher Pressepolitik nach der Revolution von 1848. Tübingen: Niemeyer.

Koszyk, Kurt (1966): Deutsche Presse im 19. Jahrhundert. Berlin: Colloquium.

Koszyk, Kurt (1989): Kommunikationsgeschichte als Sozialgeschichte. In: Winfried Schulz, Max Kaase (Hrsg.), Massenkommunikation: Theorien, Methoden, Befunde. Opladen: Westdeutscher Verlag, 46-56.

Kutsch, Arnulf (2008): Journalismus als Profession. Überlegungen zum Beginn des journalistischen Professionalisierungsprozesses in Deutschland am Anfang des 20. Jahrhunderts. In: Astrid Blome, Holger Böning (Hrsg.), Presse und Geschichte. Leistungen und Perspektiven der historischen Presseforschung. Bremen: edition lumière, 289-325. 
Lassalle, Ferdinand (1919 [1863]): Die Feste, die Presse und der Frankfurter Abgeordnetentag. In: Ferdinand Lassalle: Gesammelte Reden und Schriften, 3. Band: Die Agitation für den Allgemeinen deutschen Arbeiterverein. Das Jahr 1863. Berlin: Paul Cassirer, 339-400.

Lassalle, Ferdinand (1984 [1863]): Die Presse. Ein Symptom des öffentlichen Geistes. In: Jürgen Wilke (Hrsg.), Pressefreiheit, Darmstadt: Wissenschaftliche Buchgesellschaft, 222-238.

Löbl, Emil (1903): Kultur und Presse. Leipzig: Duncker \& Humboldt.

Luhmann, Niklas (2005 [1987]): Die Ausdifferenzierung von Politik und Recht und ihre gesellschaftlichen Grundlagen, In: ders.: Soziologische Aufklärung 4. Beiträge zur funktionalen Differenzierung der Gesellschaft, 3. Auflage. Wiesbaden: VS, 33-50.

Maase, Kaspar (1997): Grenzenloses Vergnügen. Der Aufstieg der Massenkultur 1850-1970. Frankfurt am Main: Fischer.

Malik, Maja (2004): Journalismusjournalismus. Wiesbaden: VS.

Marx, Karl; Engels, Friedrich (1969): Pressefreiheit und Zensur. Herausgegeben und eingeleitet von Iring Fetscher. Frankfurt am Main u. a.: Europäische Verlags-Anstalt.

Marx, Karl; Engels, Friedrich (1975): Gesamtausgabe (MEGA). 1. Band. Berlin: Dietz.

Mayring, Philipp (2008): Qualitative Inhaltsanalyse. Grundlagen und Techniken. 10. Auflage. Weinheim/Basel: Beltz.

Meier, Klaus (2002): Ressort, Sparte, Team. Wahrnehmungsstrukturen und Redaktionsorganisation im Zeitungsjournalismus. Konstanz: UVK.

Meier, Klaus (2005): Redaktion. In: Siegfried Weischenberg, Hans J. Kleinsteuber, Bernhard Pörksen (Hrsg.), Handbuch Journalismus und Medien, Konstanz: UVK, 394-398.

Müchler, Günter (1998): Wie ein treuer Spiegel. Die Geschichte der Cotta'schen Allgemeinen Zeitung. Darmstadt: Wissenschaftliche Buchgesellschaft.

Naujoks, Eberhard (1975): Die parlamentarische Entstehung des Reichspressegesetzes in der Bismarckzeit (1848/74). Düsseldorf: Droste Verlag.

Pörksen, Bernhard (2006): Die Beobachtung des Beobachters. Konstanz: UVK.

Pöttker, Horst (2000): Heines Tagesberichte für die ,Allgemeine Zeitung’. Ein Beitrag zu Geschichte und Bestimmung der Reportage. In: Otfried Jarren, Gerd G. Kopper, Gabriele Toepser-Ziegert (Hrsg.), Zeitung - Medium mit Vergangenheit und Zukunft. München: K.G. Saur, 27-46.

Pöttker, Horst (2003): Nachrichten und ihre kommunikative Qualität. Die „Umgekehrte Pyramide“. Ursprung und Durchsetzung eines journalistischen Standards. In: Publizistik 48, Nr. 4, 414-426.

Pöttker, Horst (2005a): The News Pyramid and It's Origins from the American Journalism in the 19th Century. A Professional Approach and an Empirical Inquiry. In: Svennik Høyer, Horst Pöttker (Hrsg.), Diffusion of the News Paradigm 1850-2000. Göteburg: Nordicom, 51-64.

Pöttker, Horst (2005b): Nachrichtenpyramide setzte sich um 1880 durch. Als die Zeitungsverleger das Publikum entdeckten. In: Journalistik Journal, 8/1, 20-21.

Pöttker, Horst (2008): Modell Heinrich Heine. Über das Verhältnis von Journalismus und Schriftstellertum in Deutschland. In: Oliver Hahn, Roland Schröder (Hrsg), Journalistische Kulturen. Internationale und interdisziplinäre Theoriebausteine. Köln: Halem, 56-75.

Pürer, Heinz (2003): Publizistik und Kommunikationswissenschaft. Ein Handbuch. Konstanz: UVK.

Pürer, Heinz, Raabe, Johannes (2002): Zur Berufsgeschichte des Journalismus. In: Irene Neverla, Elke Grittmann, Monika Pater (Hrsg.), Grundlagentexte zur Journalistik. Konstanz: UVK, 408-416.

Rabe, Horst (1991): Deutsche Geschichte 1500-1600. Das Jahrhundert der Glaubensspaltung. München: C. H. Beck.

Requate, Jörg (1995): Journalismus als Beruf. Entstehung und Entwicklung des Journalistenberufs im 19. Jahrhundert, Deutschland im internationalen Vergleich. Göttingen: Vandenhoeck \& Ruprecht.

Requate, Jörg (2008): Gescheiterte Existenzen? Zur Geschichte des Journalistenberufs im 19. Jahrhundert. In: Martin Welke, Jürgen Wilke (Hrsg.), 400 Jahre Zeitung. Die Entwicklung der Tagespresse im internationalen Kontext. Bremen: edition lumière, 335-354. 
Retallack, James (1993): From Pariah to Profession? The Journalist in German Society and Politics, from the late Enlightment to the Rise of Hitler. In: German Studies Review 16, 175-223.

Rühl, Manfred (1980): Journalismus und Gesellschaft. Bestandsaufnahme und Theorieentwurf. Mainz: Hase \& Koehler.

Rühl, Manfred (1992): Theorie des Journalismus. In: Roland Burkart, Walter Hömberg (Hrsg.), Kommunikationstheorien. Ein Textbuch zur Einführung. Wien: Braumüller, 117-133.

Schneider, Franz (1966): Pressefreiheit und politische Öffentlichkeit. Studien zur politischen Geschichte Deutschlands bis 1848. Neuwied: Luchterhand.

Schneider, Wolf; Esslinger, Detlef (2007): Die Überschrift: Sachzwänge, Fallstricke, Versuchungen, Rezepte. Berlin: Econ.

Schudson, Michael (1991): Preparing the Minds of the People: Three Hundred Years of the American Newspaper. In: John B. Hench (Ed.), Three Hundred Years of the American Newspaper. Worchester: American Antiquarium Society, 421-443.

Stöber, Rudolf (1994): Der Prototyp der deutschen Massenpresse. Der „Berliner Lokal-Anzeiger" und sein Blattmacher Hugo von Kupffer. In: Publizistik 39/3, S. 314-330.

Stöber, Rudolf (2005a): Apriori des Wandels oder „Agents of Change”? In: Markus Behmer, Bernd Blöbaum, Armin Scholl, Rudolf Stöber (Hrsg.), Journalismus und Wandel: Analysedimensionen, Konzepte, Fallstudien. Wiesbaden: VS, 13-40.

Stöber, Rudolf (2005b): Deutsche Pressegeschichte. Konstanz: UVK.

Stöber, Rudolf (2008): Kommunikations- und Medienwissenschaften. Eine Einführung. München: Beck.

Stoklossa, Paul (1910): Der Arbeitsmarkt der Redakteure. In: Jahrbücher für Nationalökonomie und Statistik 3/4, 531-535.

Streißler, Friedrich (1912): Der Schriftsteller und Journalist. Eine Darstellung des Werdeganges, der Bildungsmöglichkeiten, des Erwerbs und der Aussichten in literarischen Berufen. Stuttgart: Verlag von Wilhelm Biolet.

Verlag der Frankfurter Zeitung (Hrsg.) (1911): Die Geschichte der Frankfurter Zeitung - Volksausgabe, Frankfurt am Main: Verlag der Frankfurter Zeitung.

Weber, Johannes (2005): Straßburg 1605: Die Geburt der Zeitung. In: Jahrbuch für Kommunikationsgeschichte. 7. Band. Stuttgart: Steiner, 3-26.

Wehle, Johann Hermann (1883): Die Zeitung. Ihre Organisation und Technik. Journalistisches Handbuch. Wien/Pelt/Leipzig: A. Hartleben's Verlag.

Wehler, Hans-Ulrich (1987a): Deutsche Gesellschaftsgeschichte, 1. Band: Vom Feudalismus des Alten Reiches bis zur Defensiven Modernisierung der Reformära 1700-1815. München: Beck.

Wehler, Hans-Ulrich (1987b): Deutsche Gesellschaftsgeschichte, 2. Band: Von der Reformära bis zur industriellen und politischen „Deutschen Doppelrevolution“ 1815-1845/49, Frankfurt am Main: Büchergilde Gutenberg.

Wehler, Hans-Ulrich (1995): Deutsche Gesellschaftsgeschichte, 3. Band: Von der „Deutschen Doppelrevolution“ bis zum Beginn des Ersten Weltkrieges 1849-1914. München: Beck.

Weinhold, Kurt (1969): Die Geschichte eines Zeitungshauses 1620-1945. Köln: M. DuMont Schauberg.

Weischenberg, Siegfried (1978 [1976]): Die Außenseiter der Redaktion: Struktur, Funktion und Bedingungen des Sportjournalismus. Theorie und Analyse im Rahmen eines allgemeinen Konzepts komplexer Kommunikatorforschung. 2. Auflage. Bochum: Brockmeyer.

Weischenberg, Siegfried (2001): Nachrichtenjournalismus, Wiesbaden: Westdeutscher Verlag

Weischenberg, Siegfried (2004 [1992]): Journalistik. 1. Band. Wiesbaden: Westdeutscher Verlag.

Weischenberg, Siegfried (2005): Journalismus. In: Siegfried Weischenberg, Hans J. Kleinsteuber, Bernhard Pörksen (Hrsg.), Handbuch Journalismus und Medien. Konstanz: UVK, 132-142.

Weischenberg, Siegfried; Birkner, Thomas (2008): News Story. In: Wolfgang Donsbach (Ed.), The International Encyclopedia of Communication. 7. Band. Oxford, UK/Malden, MA: WileyBlackwell, 3277-3281.

Weischenberg, Siegfried; Malik, Maja; Scholl, Armin (2006): Souffleure der Mediengesellschaft. Report über die Journalisten in Deutschland. Konstanz: UVK.

Welke, Martin (2008): Johann Carolus und der Beginn der periodischen Tagespresse. Versuch, einen Irrweg der Forschung zu korrigieren. In: Martin Welke, Jürgen Wilke (Hrsg.), 400 Jahre Zei- 
tung. Die Entwicklung der Tagespresse im internationalen Kontext. Bremen: edition lumière, 9-122.

Wetzel, Hans-Wolfgang (1975): Presseinnenpolitik im Bismarckreich (1874-1890). Das Problem der Repression oppositioneller Zeitungen. Bern/Frankfurt am Main: Lang.

Wilke, Jürgen (1984): Nachrichtenauswahl und Medienrealität in vier Jahrhunderten. Eine Modellstudie zur Verbindung von historischer und empirischer Publizistikwissenschaft. Berlin/New York: Walter de Gruyter.

Wilke, Jürgen (2000): Grundzüge der Medien- und Kommunikationsgeschichte. Von den Anfängen bis ins 20. Jahrhundert. Köln/Weimar/Wien: Böhlau.

Wilke, Jürgen (2001): Auf dem Weg zur journalistischen Nachricht? Wer, Was, Wann, Wo, Wie, Warum, Woher in historischer Tradition. In: Dieter Möhn, Dieter Roß, Marita Tjarks-Sobhani (Hrsg.), Mediensprache und Medienlinguistik. Frankfurt am Main: Lang, 19-30.

Wilke, Jürgen (2002): Unter Druck gesetzt. Vier Kapitel deutscher Pressegeschichte. Köln/Weimar/ Wien: Böhlau.

Wilke, Jürgen (2003): The History and Culture of the Newsroom in Germany. In: Journalism Studies 4/4, 465-477.

Wischermann, Ulla (1983): Frauenfrage und Presse. Frauenarbeit und Frauenbewegung in der illustrierten Presse des 19. Jahrhunderts. München/New York/London/Paris: K. G. Saur.

Wischermann, Ulla (1996): Quellen und Methoden historischer Rezeptionsforschung. In: Gudrun Marci-Boehncke, Petra Werner (Hrsg.): BlickRichtung Frauen. Theorien und Methoden geschlechtsspezifischer Rezeptionsforschung. Weinheim: Deutscher Studien Verlag, S. 133-146.

Wolter, Hans-Wolfgang (1981): Generalanzeiger - das pragmatische Prinzip. Zur Entwicklungsgeschichte und Typologie des Pressewesens im späten 19. Jahrhundert mit einer Studie über die Zeitungsunternehmungen Wilhelm Girardets (1838-1918). Bochum: Studienverlag Dr. N. Brockmeyer.

Wrede, Richard (1906 [1902]): Handbuch der Journalistik. Berlin: Verlag Dr. R. Wrede. 